Damage threshold - Summary

\title{
Laser induced damage threshold testing
}

Today's latest laser technologies can be used for a diverse array of applications. Currently, however, their use is being hindered by a lack of knowledge about certain
power thresholds, above which power thresholds, above which lasers can damage valuable Through their research Dr Jan Vanda and colleagues at the HiLASE Centre, part of the Institute of Physics at the Czech Academy of Sciences pushes accuracy of established schemes and introduces new testing procedres to accur tely test variety of materials. Their approach enables research and manufacturers alike to better exploit the advanced capabilities of modern high power lasers.

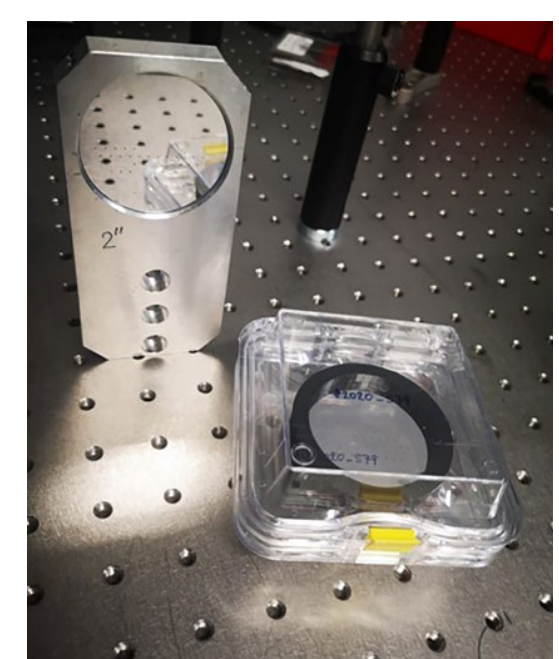

ver recent decades, laser
technologies have advanced
a at a breakneck pace. Today, even commercially available lasers are able to produce high intensity beams which would have been unthinkable just a few years ago, and can focus the precision. Thanks to these advances, precision. Thanks to hise advances, laser equipment including cuttingedge facilities like HiLASE in the Czech Republic.

Today, high power laser systems are being used in a wide variety of research applications, from aeronautic, automotive, biomed and pharma, for 3D printing, power generation and tooling, and towards material physics. In particular, they are critical for studies involving complex arrangements of optics, where beams must be fired alon precisely defined directions, often used at laser particles acceleration facilities. Elsewhere, lasers are increasingly being used for industrial applications, where cutting, drilling, and surface texturing to

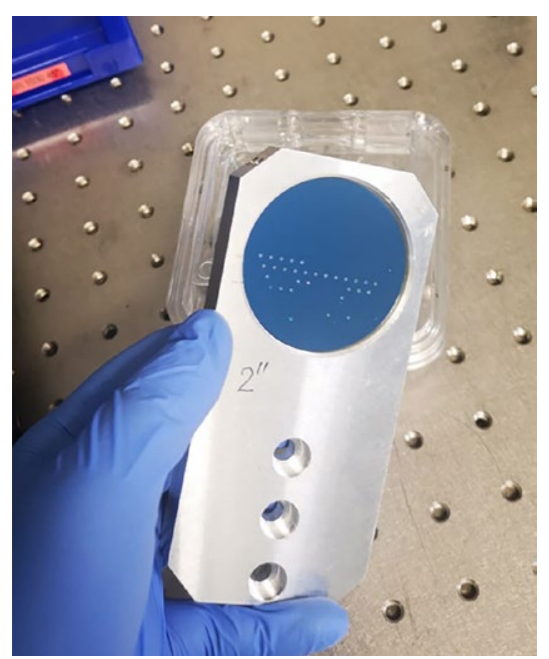

be carried out with extreme degrees of precision on a variety of materials.

Unfortunately, today's most advanced lasers are presenting a novel problem to the many groups which benefit trom to damage from lasers, it can be to damage from lasers, it can be

and developers to determine how

far they can stretch their capabilities.

"Increasingly powerful lasers are

now being built to meet the needs of evolving technologies," says Dr Vanda. "Laser damage is a key parameter for all components in any high-power laser system, establishing limits of maximum achievable energy in a laser beam."

Since these limits can vary widely between different materials and laser parameters, there is no easy way for researchers and manufacturers to accurately define where they lie. Withou heir lasers carefully, must operate maximum possible well below their maximum possible energles, where they own components. This problem places a stringent limit on the maximum possible energies at which lasers can operate.

\section{QUANTIFYING DAMAGE}

THRESHOLDS

, a set of standards Organisation for Standardisation (ISO) in 2011. "By the ISO 21254 standard, the LIDT is defined as highest quantity of for for whe to enure stabling ans. "Therefore, high-power laser, it is neliability of any the means to accur tely tha aser induced damage threshold (or LIDT)."

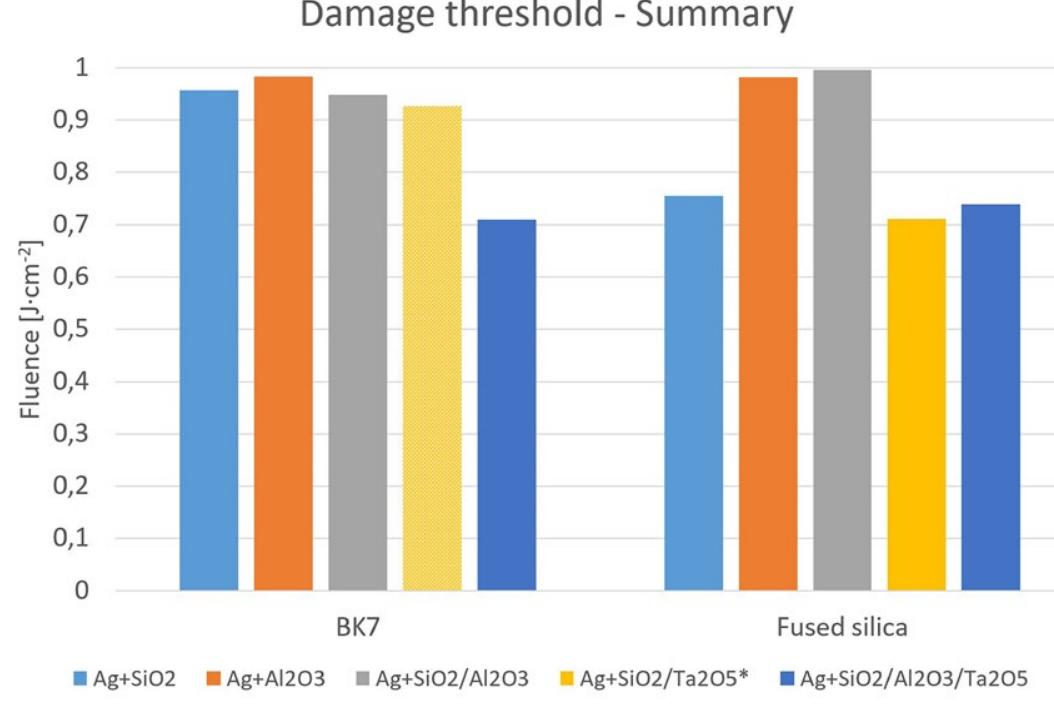

Vanda et. al., SPIE Laser Damage 2020. Laser damage performance of hybrid mirrors

Laser damage is a key parameter for all components in any high-power laser system, establishing limits of maximum achievable energy in a laser beam.

Depending on environmental

conditions, the LIDT can vary widely based on optical components selection and laser operation parameters. Within the interiors of samples, imperfections and defects at molecular and atomic levels will often result from ageing, conditioning, and environmental wear. It they are exposed to laser beams with sufficiently high energies, these features can induce interference in laser light scales, all of these features can strongly enhance the strengths of the electric fields carried by laser beams.

Comparable effects can also be found on material surfaces, which feature imperfections including roughness, optical absorption, and the presence of contaminating particles; all of which can induce destructive local heating. interference and other disruptive effects. If any of these factors are not fully accounted for in LIDT evaluation laser operators risk inflicting potentially irreversible damage on ISO systems, even if they adhere to sis standards. For Dr Vanda's team, determining LDTs in specific mat

and conditions. To do this, the researchers have developed advance damage at HiLAS

MEASUREMENTS AT HILAS Situated just outside Prague, HiLASE is home to two state-of-the-art laser and Perla. While the two systems produce light in different ways, they both operate by extremely high peak powers. When these pulses are directed onto materia samples, in conditions where the humidity, temperature, and dust particle concentration is tightly controlled, their high energies enable the LIDT to be measured using laser beams with large diameters. Compared with previous studies, this allows DrVanda's team to calculate damage thresholds over larger areas - significantly reducing errors in their results. Further, such laser sources enable more options when designing aser damage tests for particular areas and applications.

"At HiLASE, we are aiming to develop "At un ming encrias and overall feasibility of developed laser systems. These efforts have led HiLASE to construct and operate some of the brightest lasers in the world."

Silver layer degradation issues

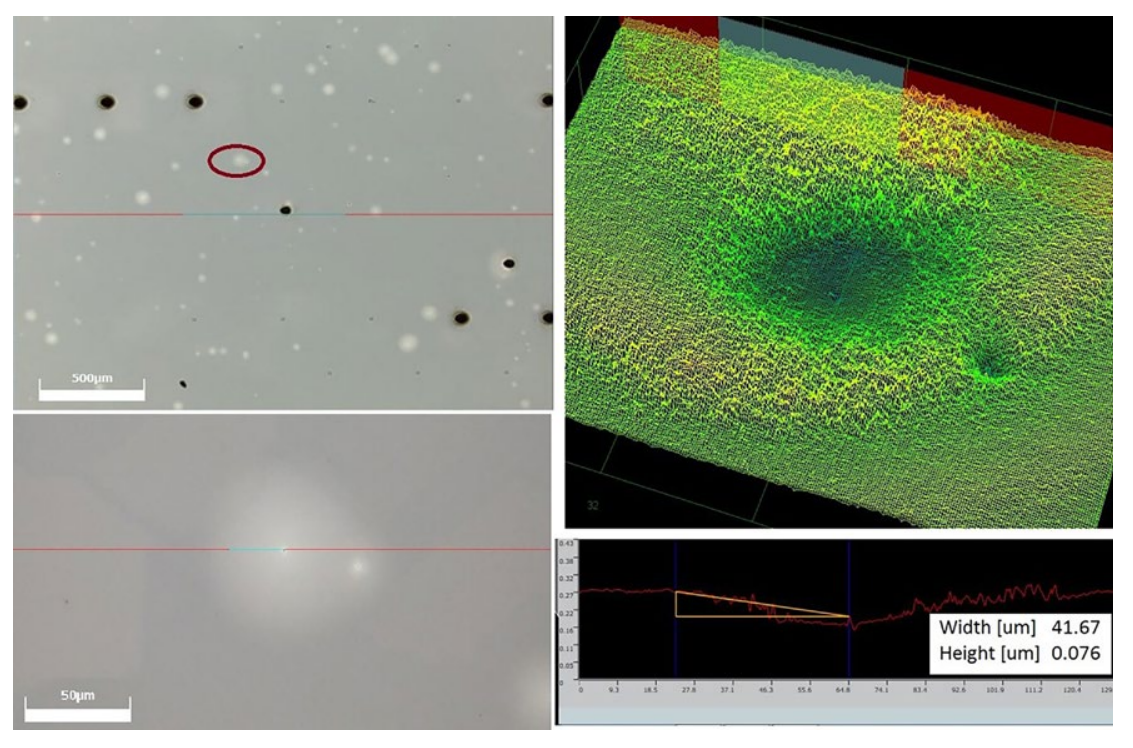

To ensure stability and reliability of any high-power laser, it is necessary to have a means to
accurately determine the laser induced damage threshold (IDT) 


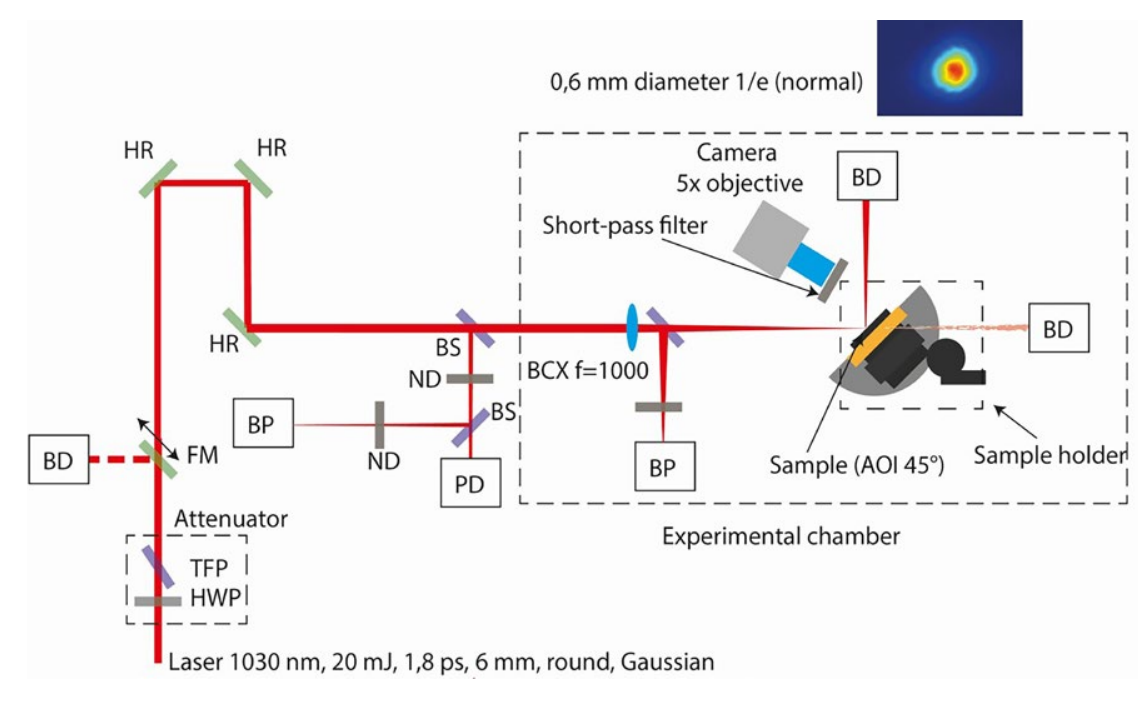

Test scheme: Laser induced damage threshold (LIDT) testing station.

anti-reflective surfaces and hybrid mirrors. These hybrid mirrors, a multistack divectric repped with a typical example of HiLASE's IDT testing approach. Although such components combine advantages of metallic and dielectric mirrors, their behaviour is not easily predictable, in particular when used with highenergy, large-aperture laser beam.

Since even highly reflective dielectric mirrors must always absorb some light, and metallic coatings are susceptible to the heat deposition generated by absorption, it is particularly important for researchers to measure their damage thresholds at different exposition conditions.

successfully determin the LIDT value for hybrid coatings in the picosecond range for the first time and address the issues of different techniques used for coatings deposition.

In another study, Dr Vanda's team demonstrated HiLASE's LIDT testing capabilities by measuring values in nanosecond regime for both highlyand anti-reflective coatings when they were deposited onto three different substrates. YAG, fused silica and BK7 glass. Samples were selected to demonstrate testing capabilities for novel $\mathrm{ZrO}_{2}$-based coatings deposite by so-called IAD technique,

where LIDT values were used as a the efficacy of the dever

coating process. In each case, the ies enabled the for all arrangements of substralues coating to unprecedented degrees of accuracy.

IMPROVING PERFORMANCE IN REAL APPLICATIONS

Through their continuing experiments, the results gathered by DrVanda's areas, we were aberent application reas, including efficient coating removal, laser material processing interference patterning, or fibre beam delivery."

Such advanced techniques would greatly simplify a variety of common tasks. For industrial manufacturing, these could include the replacement of aged or damaged coatings, which are often firmly adhered to material surfaces. While laser-based processes for removing these coatings often risk harming the material underneath, the team's testing techniques enable delivery of just the right amount flaser power, without any risk of interference patterns between crossing laser beams, manufacture could also ablate out specific repeating patterns onto materia surfaces, enabling control over properties as frictions, wettability or biocompatibility. Users with the knowledge of material damage behaviour would be able to optimise

The results gathered by Dr Vanda"s team could soon provide valuable insights into how lasers should be operated in many everyday situations.

insights into how lasers should be operated in many everyday situation. previously hindered by were in the laser energies at which damage occurs, the testing facilities at HiLASE provide long-awaited solutions. As Dr Vanda concludes, "Utilising our

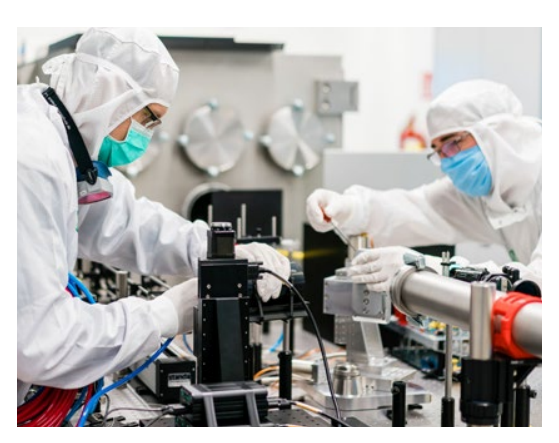

their processes towards higher yield and efficiency. Furthermore, operators may map out the elevation profiles of rough surfaces, and steer the paths of laser beams by directing them through optical fibres, along with a wide array of other potential uses.

Alongside these myriad industrial applications, the team's approach could enable researchers to operate experiments involving complex optical setups more safely. With the reassurance that no costly damages oxperinental components win fin occur, these researchers would finally their highest possible brightners which wound ht possible brightness too risky only a few years ago.

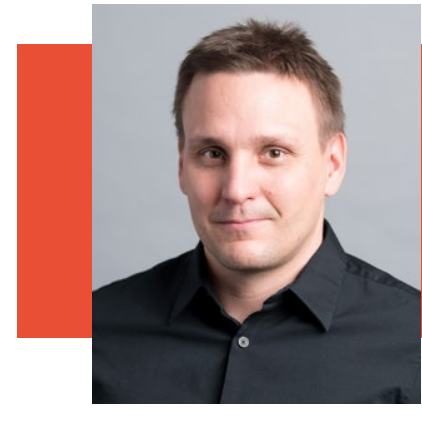

\section{Behind the Research} Dr Jan Vanda

: jan.vanda@hilase.cz T: $+420314007747 \quad$ W: www.hilase.cz
https://www.linkedin.com/in/jan-vanda-b6801623/

Research Objectives

Jan Vanda's research provides laser induced damage threshold (LIDT) testing at the highest technical level.

\section{Detail}

Jan Vanda

Institute of Physics CAS

Za Radnici 828

25241 Dolni Brezany

Czech Republic, www.hilase.cz

Bio

Jan Vanda received his PhD degree in Optoelectronics from the Technical University of Ostrava, Czech Republic. He completed a one-year Marie-Curie fellowship as post-doc at FORTH-IESL Crete, where he researched microstructured fibres and superparamagnetic nanoparticles. He then joined Czech Office for Standards as a national secretary CEN Wenational standardisation organisations IEC, CENELEC and ETSI. In 2011 Dr Vanda joined the the HiLASE Price CAS, where he paricipated phenomena. He is currently head of the LIDT laboratory at HiLASE Centre, Czech Republic.

\section{Collaborators}

- Tomas Mocek, Ph. D.
- Jan Brajer, Ph.D.

- Mihai-George Muresan, Ph.D.

- Pavel Cech

- Martin Mydlar

- Matterina Pilna

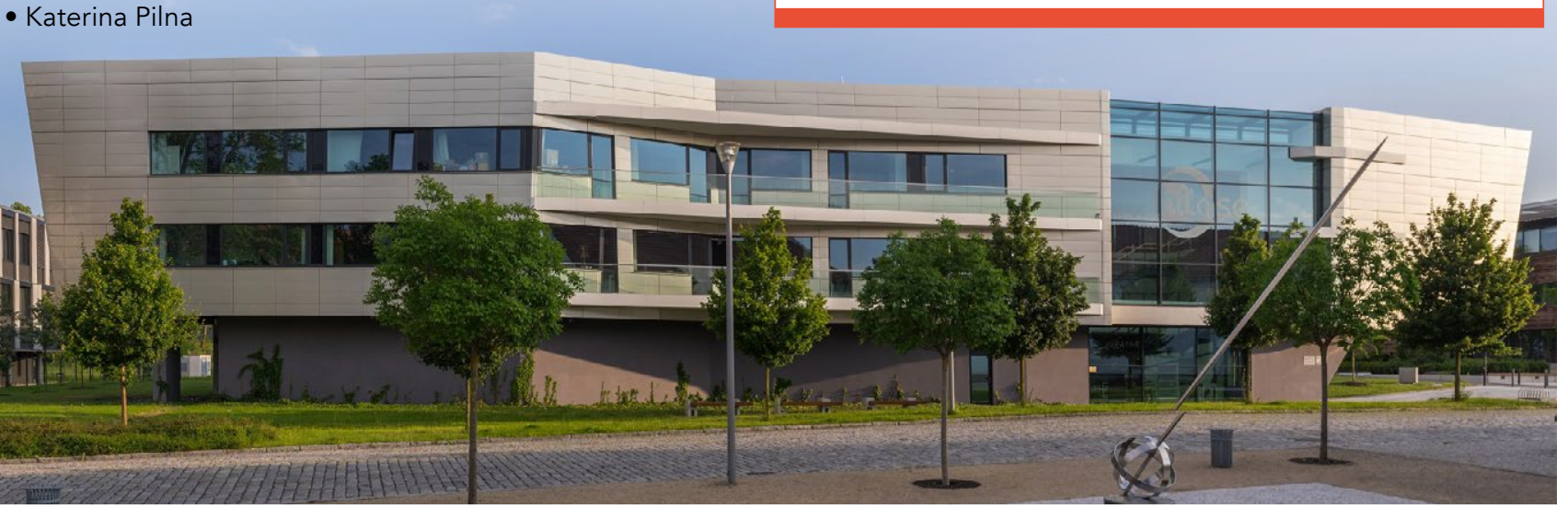
(2019). Laser Induced Damage Threshold Testing at HiLASE, MM Science Journal, 3657-3661.

Vanda, J., Muresan, M., Cech, P., Mydlár, M., Pilna, K., Brajer, J., Uxa, S., Skoda, V. (2020, September). Multiple pulse picosecond laser induced damage threshold on Materials, 11514, 115141C. International Society for Optics and Photonics.

\section{Personal Response}

\section{How easy will it be for other laser facilities to} replicate your testing approach?

This is actually quite a tricky question. In theory it approach could be transferred anywhere as However, this approach benefits from long-term experience in the field, combined with our unique background of developing state-of-the-art laser systems.

Our target is to take research principles from the scientific laboratory and transfer them towards real task. We have an established team of highly educated, skilled, devoted and enthusiastic Nevertheless, we are here and always keen to help
Cech, P., Vanda, J., Muresan, M G., Mydlar, K P., Brajer, J. 